\title{
Geometric Curvatures of Plane Symmetry Black Hole
}

\author{
Shao-Wen Wei, Yu-Xiao Liu, Chun-E. Fu, and Hai-Tao Li
}

Institute of Theoretical Physics, Lanzhou University, Lanzhou 730000, China

Correspondence should be addressed to Yu-Xiao Liu; liuyx@lzu.edu.cn

Received 16 February 2013; Accepted 23 May 2013

Academic Editor: Hernando Quevedo

Copyright (C) 2013 Shao-Wen Wei et al. This is an open access article distributed under the Creative Commons Attribution License, which permits unrestricted use, distribution, and reproduction in any medium, provided the original work is properly cited.

\begin{abstract}
We study the properties and thermodynamic stability of the plane symmetry black hole from the viewpoint of geometry. We find that the Weinhold curvature gives the first-order phase transition at $N=1$, where $N$ is a parameter of the plane symmetry black hole while the Ruppeiner one shows first-order phase transition points for arbitrary $N \neq 1$. Considering the Legendre invariant proposed by Quevedo et al., we obtain a unified geometry metric, which contains the information of the second-order phase transition. So, the first-order and second-order phase transitions can be both reproduced from the geometry curvatures. The geometry is also found to be curved, and the scalar curvature goes to negative infinity at the Davie phase transition points beyond semiclassical approximation.
\end{abstract}

\section{Introduction}

Several decades ago, the original work of Bekenstein and Hawking showed that the black hole is indeed a thermodynamics system $[1,2]$. It was also found that the black hole satisfies four laws of the elementary thermodynamics with regarding the surface gravity and the outer horizon area as its temperature and entropy, respectively [3]. Although, it is widely believed that a black hole is a thermodynamic system, the statistical origin of the black hole entropy is still one of the most fascinating and controversial subjects today.

The investigation of thermodynamic properties of black holes is also a fascinating subject. Much of work had been carried out on the stability and phase transitions of black holes. It is generally thought that the local stability of a black hole is mainly determined by its heat capacity. Negative heat capacity usually gives a thermodynamically unstable system, and the positive one implies a local stable one. The divergent points of the heat capacity are usually consistent with the Davies points, where the second-order phase transition takes place [4-6].

The properties and phase transitions of a thermodynamic system can also be studied with the idea of geometry. Weinhold [7] first introduced the geometrical concept into the thermodynamics. He suggested that a Riemannian metric can be defined as the second derivatives of internal energy $U$ with respect to the entropy and other extensive quantities of a thermodynamic system. However, it seems that the Weinhold geometry has not much physical meanings. Few years later, Ruppeiner [8] introduced another metric, which is analogous to the Weinhold one. The thermodynamic potential of the Ruppeiner geometry is the entropy $S$ of the thermodynamic system rather than the internal energy $U$. In fact, the two metrics are conformally related to each other:

$$
d s_{R}^{2}=\frac{1}{T} d s_{W}^{2}
$$

with the temperature $T$ as the conformal factor. The Ruppeiner geometry had been used to study the ideal gas and the van der Waals gas. It was shown that the curvature vanishes for the ideal gas, whereas for the van der Waals gas, the curvature is nonzero and diverges only at those points where the phase transitions take place (for details see the review paper [9]). The black hole, as a thermodynamics system, has been extensively investigated. The Weinhold geometry and the Ruppeiner geometry were obtained for various black holes and black branes [4-30]. In particular, it was found that the Ruppeiner geometry carries the information of phase structure of a thermodynamic system. In general, its curvature is singular at the points, where the phase transition takes place. However, for the Banados-Teitelboim-Zanelli (BTZ) and Reissner-Nordström (RN) black holes, the cases 
are quite different. The Ruppeiner geometries give a vanished curvature, which means there exist no thermodynamic interactions and no phase transition points. There exist phase transition points for the two kinds of black hole. For the contradiction, much research has been carried out to explain it. The main focus is on the thermodynamic potential, which is generally believed to be the internal energy $U$ rather than the mass $M$. For the Reissner-Nordström black hole, it was argued in [18] that the thermodynamic curvature should be reproduced from the Kerr-Newmann anti-de sitter black hole with the angular momentum $J \rightarrow 0$ and cosmological constant $\Lambda \rightarrow 0$. Another explanation of the contradiction was presented by Queved et al. few years ago [31, 32]. They pointed out that the origin of the contradiction is that the Weinhold metric and Ruppeiner metric are not Legendre invariants. A Legendre invariant metric was introduced by them, which could reproduce correctly the behavior of the thermodynamic interactions and second-order phase transitions for the BTZ and RN black holes $[33,34]$ and other black hole configurations and models [35-38]. Inspired by the thermodynamic geometry, Liu et al. recently proposed a free energy metric [39], which can give a better description on the phase transition for a black hole. The authors also showed that, for a system with $n$-pairs of intensive/extensive variables, different thermodynamic metrics can be embedded into a flat $(n, n)$-dimensional space. The method has been extended to different black holes [40-44].

Another interesting and important question of this field is how the geometry behaves beyond semiclassical approximation. It is generally believed that there will be a logarithmic corrected term to the entropy when the semiclassical black hole extends to its quantum level $[45,46]$. Considering the correction term, the geometry structure was studied in [33, 47] for the BTZ black hole. Especially, its Ruppeiner curvature will be nonzero beyond semiclassical approximation. The aim of this paper is to study the phase transitions and geometry structure of the plane symmetry black hole. Firstly, we study the thermodynamic stability of the plane symmetric black hole. It is shown that there always exist locally thermodynamically stable phases and unstable phases for the plane symmetric black hole due to suitable parameter regimes. Then, three different geometry structures are obtained. The Weinhold curvature gives phase transition points, which correspond to that of the first-order phase transition only at $N=1$, while the Ruppeiner one shows first-order phase transition points for arbitrary $N \neq 1$. Considering the Legendre invariant, we obtain a unified geometry metric, which gives a correct behavior of the thermodynamic interactions and phase transitions. It is found that the curvature constructed from the unified metric goes to negative infinity at the Davies' points, where the second-order phase transition takes place. The geometry structure is also studied as the logarithmic correction is included. The results show that the unified geometry behaviors differ when logarithmic correction term is included. However, it has no effect on the unified geometry depicting the phase transitions of the plane symmetric black hole.

The paper is organized as follows. In Section 2, we first review some thermodynamic quantities of the plane symmetric black hole. The thermodynamic stability is also studied. In Section 3, both the Weinhold and Ruppeiner geometry structures are obtained. However, they fail to give the information about the second-order phase transition points. For the reason, we give a detailed analysis and obtain a new Legendre invariant metric structure which could give a good description of the thermodynamic interactions and phase transitions in Section 4. Unified geometry structure beyond semiclassical approximation is also considered in Section 5. Finally, the paper ends with a brief conclusion.

\section{Thermodynamic Quantities and Thermodynamic Stability of the Plane Symmetric Black Hole}

In this section, we will present the thermodynamic quantities and other properties of the plane symmetric black hole. The local thermodynamic stability of it is also discussed. The action depicting the plane symmetric black hole is given by

$$
S=\frac{1}{16 \pi} \int d^{4} x \sqrt{-g}\left(\mathscr{R}-2(\nabla \varphi)^{2}-2 \Lambda e^{2 b \varphi}-e^{-2 a \varphi} F^{2}\right),
$$

where $\varphi$ is a dilaton field and $a, b$ are constants. The negative cosmological constant is $\Lambda=-3 \alpha^{2}$. Static plane symmetric black hole solutions in this theory were first given in [48] (some detail work for the black hole can also be found in [4953]). Consider the following:

$$
d s^{2}=-f(r) d t^{2}+f^{-1}(r) d r^{2}+g(r)\left(d x^{2}+d y^{2}\right) .
$$

The metric functions are given by, respectively,

$$
\begin{gathered}
f(r)=-\frac{4 \pi M}{N \alpha^{N}} r^{1-N}+\frac{6 \alpha^{2}}{N(2 N-1)} r^{N}+\frac{2 Q^{2}}{N \alpha^{2 N}} r^{-N}, \\
g(r)=(r \alpha)^{N} .
\end{gathered}
$$

The dilaton field $\varphi$ reads

$$
\varphi(r)=-\frac{\sqrt{2 N-N^{2}}}{2} \ln r .
$$

And the constant $a=b=\sqrt{2 N-N^{2}} / N$. From (5), one easily finds the parameter $N \in(1 / 2,2)$. The scalar curvature of this spacetime can be calculated as

$$
R=12 \alpha^{2} r^{N-2}+\frac{\left(2 N-N^{2}\right)}{2 r^{2}} f(r) .
$$

Obviously, $R$ diverges at $r=0$ for any value of $N$, which implies that $r=0$ plane is a singularity plane. From Figure 1, we see that the scalar curvature $R$ is a monotonically decreasing function of $r$ for different $N$. It is also clear that $R$ has a large value for small value of $N$ near $r=0$.

The parameters $M$ and $Q$ are the mass and charge of the black hole. The event horizon is located at $f\left(r_{h}\right)=0$ and the radius $r_{h}$ satisfies

$$
\frac{3 \alpha^{2}}{2 N-1} r_{h}^{2 N}-\frac{2 \pi M}{\alpha^{N}} r_{h}+\frac{Q^{2}}{\alpha^{2 N}}=0 .
$$




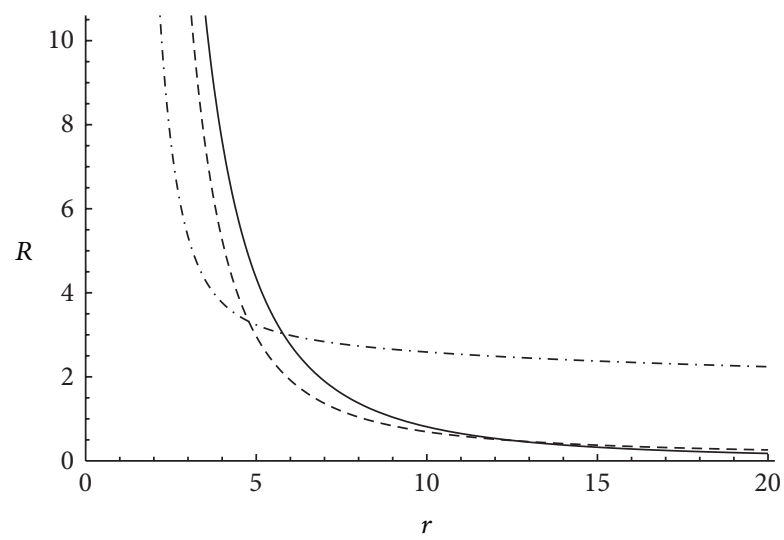

FIGURE 1: The behavior of scalar curvature $R$ with $\alpha=\sqrt{3} / 3, Q=10$, and $M=1$. The full line, dashed line, and dashed-dotted line are for $N=0.6,1$, and 1.8 , respectively.

In general, there exist two horizons, the inner horizon and the outer horizon. Under the extreme case, the two horizons will merge into one. Here, we have denoted $r_{h}$ as the radius of outer horizon. The surface area of the outer horizon corresponds to unit $x-y$ plane is [52]. One may consider the following:

$$
\mathscr{A}=\left(\alpha r_{h}\right)^{2 N} \text {. }
$$

From (7), the mass can be expressed in the form

$$
M=\frac{3 \alpha^{N+2}}{2 \pi(2 N-1)} r_{h}^{2 N-1}+\frac{Q^{2}}{2 \pi \alpha^{N}} r_{h}^{-1} .
$$

With the relation between area and entropy, that is, $S=\mathscr{A} / 4$, we can obtain

$$
r_{h}=\frac{1}{\alpha}(4 S)^{1 / 2 N}
$$

Substituting (10) into (9), the mass can be obtained as a function of entropy $S$ and charge $Q$ in the form

$$
M=\frac{3 \alpha^{3-N}}{2 \pi(2 N-1)}(4 S)^{(2 N-1) / 2 N}+\frac{Q^{2}}{2 \pi \alpha^{N-1}}(4 S)^{-1 / 2 N} .
$$

From the energy conservation law of the black hole

$$
d M=T d S+\phi d Q
$$

the relevant thermodynamic variables, the temperature, and electric potential are obtained

$$
\begin{gathered}
T=\left(\frac{\partial M}{\partial S}\right)_{Q}=\frac{\left(12 \alpha^{2} S-Q^{2}\right) \alpha^{1-N}}{2^{2+1 / N} \pi N S^{1+1 / 2 N}}, \\
\phi=\left(\frac{\partial M}{\partial Q}\right)_{S}=\frac{\alpha^{1-N} Q}{2^{1 / N} \pi S^{1 / 2 N}} .
\end{gathered}
$$

For a given charge $Q$, the heat capacity has the expression

$$
C_{Q}=-\frac{2 N S\left(12 \alpha^{2} S-Q^{2}\right)}{12 S \alpha^{2}-(1+2 N) Q^{2}},
$$

with the zeropoints and singular points

$$
\begin{gathered}
Q^{2}=12 \alpha^{2} S, \quad \text { (zero points), } \\
Q^{2}=\frac{12 \alpha^{2} S}{2 N+1}, \quad \text { (singular points), }
\end{gathered}
$$

respectively. The heat capacity $C_{Q}$ goes to zero at $Q^{2}=12 \alpha^{2} S$ continuously, which is considered to be the first-order phase transition point. On other hand, it is generally believed that the Davies' points where the second-order phase transition takes place correspond to the diverge points of heat capacity. So the heat capacity $C_{Q}$ may indicate that the second-order phase transition takes place at $Q^{2}=12 \alpha^{2} S /(2 N+1)$. The heat capacity also contains the information of the local stability of the black hole thermodynamics. The negative heat capacity always implies an unstable thermodynamics system, and the positive one shows a stable system. Here, we would like to give a brief discussion about the local stability of the plane symmetric black hole. For $Q^{2}>12 \alpha^{2} S$, the numerator of the heat capacity (14) is negative, while the denominator is positive, which gives a negative heat capacity. For $Q^{2}<12 \alpha^{2} S /(2 N+1)$, the numerator is positive, but the denominator turns to be negative, which also shows a negative heat capacity. So, in both cases, the heat capacity $C_{Q}$ implies an unstable black hole thermodynamics. However, when $|Q| \in(2 \sqrt{3} \alpha \sqrt{S /(2 N+1)}, 2 \sqrt{3} \alpha \sqrt{S})$, both the numerator and denominator are positive, which implies a stable black hole thermodynamics. The behavior of the heat capacity $C_{Q}$ can be directly found from Figure 2 . For larger and smaller values of $|Q|$, the heat capacity $C_{Q}$ is negative. While in the middle zone, it is positive, which means that the black hole can be in stable thermal equilibrium with an arbitrary volume heat bath. In summary, we have found that there always exist locally thermodynamically stable phases and unstable phases for the plane symmetric black hole due to suitable parameter regimes.

\section{Weinhold Geometry and Ruppeiner Geometry of the Plane Symmetric Black Hole}

In this section, we would like to study the Weinhold and Ruppeiner geometries of the plane symmetric black hole. In the first step, we will calculate the Weinhold geometry. Then, using the conformal relation, we could obtain the Ruppeiner geometry naturally. The Weinhold geometry is charactered by the metric

$$
d s_{W}^{2}=\frac{\partial^{2} M}{\partial S^{2}} d S^{2}+2 \frac{\partial^{2} M}{\partial S \partial Q} d S d Q+\frac{\partial^{2} M}{\partial Q^{2}} d Q^{2},
$$

where the index $W$ denotes the Weinhold geometry. Here, the thermodynamic potential is the mass $M$, and the entropy $S$ and charge $Q$ are the extensive variables. 


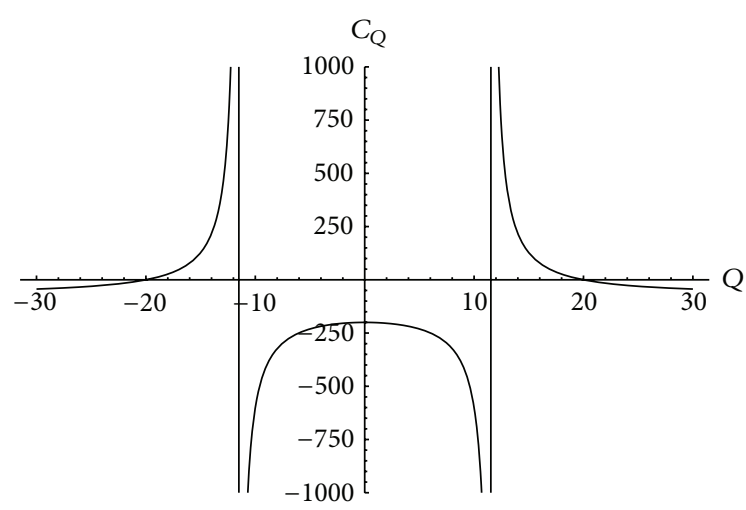

Figure 2: The behavior of the heat capacity $C_{Q}$, with $N=1, \alpha=$ $\sqrt{3} / 3$, and $S=100 . C_{Q}$ is singular at $Q= \pm 11.5470$ and vanishes at $Q= \pm 20$. form

Using (11), the Weinhold metric can be obtained in the

$$
g_{W}=-\frac{\alpha^{1-N}}{2^{1 / N} S^{1 / 2 N}}\left(\begin{array}{cc}
\frac{12 \alpha^{2} S-(2 N+1) Q^{2}}{8 \pi N^{2} S^{2}} & \frac{Q}{2 \pi N S} \\
\frac{Q}{2 \pi N S} & -\frac{1}{\pi}
\end{array}\right) .
$$

Its determinant is $\operatorname{det}(g)=-\alpha^{2-2 N}\left[12 \alpha^{2} S-(2 N-1) Q^{2}\right] /$ $2^{3+2 / N} S^{2+1 / N} \pi^{2} N^{2}$. Note that the determinant disappears as the heat capacity vanishes only at $N=1$. A simple calculation shows that the Christoffel symbols are

$$
\begin{gathered}
\Gamma_{S S}^{S}=-\frac{(2 N+1)\left[12 \alpha^{2} S-(4 N-1) Q^{2}\right]}{4 N S\left[12 \alpha^{2} S-(2 N-1) Q^{2}\right]}, \\
\Gamma_{\mathrm{QS}}^{S}=\Gamma_{S Q}^{S}=-\frac{2 N Q}{12 \alpha^{2} S-(2 N-1) Q^{2}}, \\
\Gamma_{\mathrm{QQ}}^{S}=-\frac{2 N S}{12 \alpha^{2} S-(2 N-1) Q^{2}}, \\
\Gamma_{S S}^{\mathrm{Q}}=-\frac{(2 N+1) Q^{3}}{4 N S^{2}\left[12 \alpha^{2} S-(2 N-1) Q^{2}\right]}, \\
\Gamma_{S Q}^{\mathrm{Q}}=\Gamma_{\mathrm{QS}}^{\mathrm{Q}}=-\frac{12 \alpha^{2} S+(2 N+1) Q^{2}}{4 S\left[12 N \alpha^{2} S-N(2 N-1) Q^{2}\right]}, \\
\Gamma_{\mathrm{QQ}}^{\mathrm{Q}}=\frac{\mathrm{Q}}{12 \alpha^{2} S-(2 N-1) Q^{2}},
\end{gathered}
$$

where the Christoffel symbols are calculated with

$$
\Gamma_{\mu \nu}^{\lambda}=\frac{1}{2} g^{\lambda \tau}\left(g_{\nu \tau, \mu}+g_{\mu \tau, \nu}-g_{\mu \nu, \tau}\right) .
$$

The Riemannian curvature tensor, Ricc curvature, and scalar curvature are given, respectively,

$$
\begin{gathered}
R_{\sigma \nu \tau}^{\mu}=\Gamma_{\sigma \nu, \tau}^{\mu}-\Gamma_{\sigma \tau, \nu}^{\mu}+\Gamma_{\lambda, \tau}^{\mu} \Gamma_{\sigma, v}^{\lambda}-\Gamma_{\lambda, \nu}^{\mu} \Gamma_{\sigma, \tau}^{\lambda}, \\
R_{\mu \nu}=R_{\mu \lambda \nu}^{\lambda}, \\
R=g^{\mu \nu} R_{\mu \nu} .
\end{gathered}
$$

With (21), we get the scalar curvature

$$
\mathscr{R}_{W}=-\frac{242^{1 / N} N \pi S^{1+1 / 2 N} \alpha^{1+N}}{\left[12 \alpha^{2} S-(2 N-1) Q^{2}\right]^{2}} .
$$

This curvature is always negative for any values of charge $Q$ and positive entropy $S$. It also diverges at $Q^{2}=12 \alpha^{2} S /(2 N+$ 1 ), which consists with the first-order transition points (15) reproduced from the capacity $C_{\mathrm{Q}}$ only at $N=1$. Its behavior can be seen in Figure 3. However, it implies no information about the second-order phase transition. So, it is natural to ask how the Ruppeiner curvature behaves. Could it give the proper phase transition points?

With that question, we now turn to the Ruppeiner geometry of the plane symmetric black hole. Recalling the conformal relation (1) between the Ruppeiner geometry and the Weinhold geometry, we obtain the Ruppeiner metric

$$
g_{R}=\frac{1}{T} g_{W}=\left(\begin{array}{cc}
-\frac{12 \alpha^{2} S-(2 N+1) Q^{2}}{2 N S\left(12 \alpha^{2} S-Q^{2}\right)} & -\frac{2 Q}{12 \alpha^{2} S-Q^{2}} \\
-\frac{2 Q}{12 \alpha^{2} S-Q^{2}} & -\frac{4 N S}{12 \alpha^{2} S-Q^{2}}
\end{array}\right),
$$

where the index $R$ denotes the Ruppeiner geometry. After some calculations, we obtain the Ruppeiner curvature

$$
\mathscr{R}_{R}=-\frac{12 \alpha^{2} Q^{2}(N-1)\left[36 S \alpha^{2}-(4 N-1) Q^{2}\right]}{\left(12 \alpha^{2} S-Q^{2}\right)\left[12 S \alpha^{2}-(2 N-1) Q^{2}\right]^{2}} .
$$

It is obvious that the curvature will be zero at $N=1$. The vanished thermodynamic curvature $\mathscr{R}_{R}$ implies that there exist no phase transition points and no thermodynamic interactions. So, the Ruppeiner curvature is not proper to describe the phase transitions of the plane symmetric black hole at $N=1$. The divergence of the Ruppeiner curvature is at $Q^{2}=12 \alpha^{2} S /(2 N+1)$ and $Q^{2}=12 \alpha^{2} S$, which can be seen from Figure 4 . The points $Q^{2}=12 \alpha^{2} S$ consist with the zero-points (15) of the heat capacity $C_{Q}$. This means that the Ruppeiner curvature always implies the first-order phase transition points. Like the Weinhold curvature, the Ruppeiner curvature also implies no any information about the second-order phase transition.

\section{Unified Geometry of the Plane Symmetric Black Hole}

In the previous section, we show that the Weinhold curvature implies the first-order phase transition points only at $N=1$, while the Ruppeiner curvature implies the first-order phase transition points except $N=1$. Both of the geometry structures gave no information about the second-order phase transition points of the plane symmetric black hole. Quevedo pointed out that the two geometries are not Legendre invariants, which makes them inappropriate to describe the geometry of thermodynamic systems [34]. Considering the Legendre invariant, a unified geometry was presented in [35], where the metric structure can give a good description 


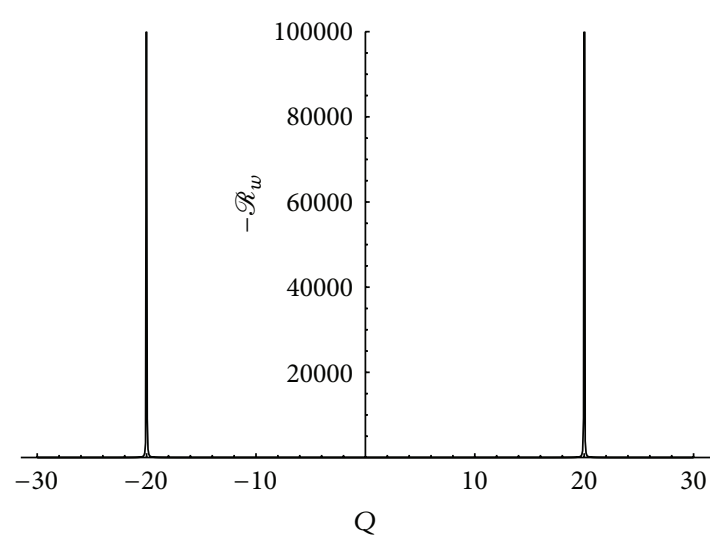

Figure 3: The negative Weinhold curvature $\mathscr{R}_{w}$ versus the charge $Q$ with $N=1, \alpha=\sqrt{3} / 3$, and $S=100$. The divergence points are at $Q= \pm 20$.

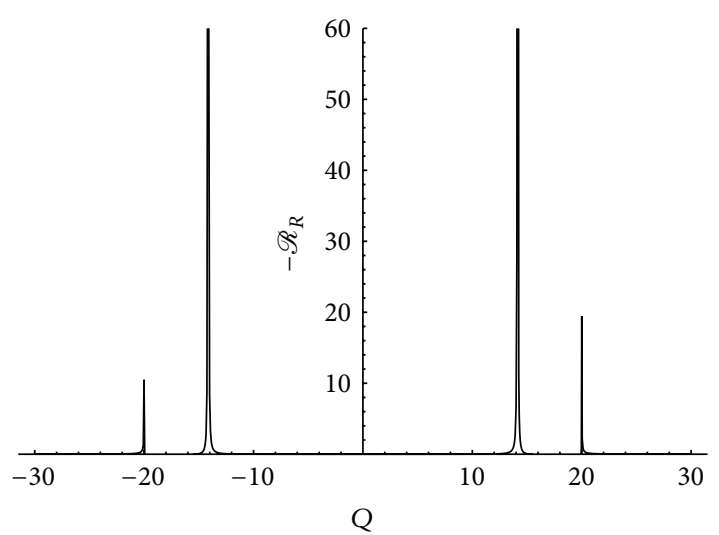

FIgURE 4: The negative Ruppeiner curvature $\mathscr{R}_{R}$ versus the charge $Q$ with $N=3 / 2, \alpha=\sqrt{3} / 3$, and $S=100$. The divergence points are at $Q= \pm 20, \pm 14.1421$.

of various types of black hole thermodynamics. So, in this section, we would like to discuss the unified geometry of the plane symmetric black hole, and we want to know whether it works.

Here, we still take the mass $M$ as the thermodynamic potential. Then the unified geometry metric can be expressed as

$$
\begin{aligned}
d s_{L}^{2}= & \left(S \frac{\partial M}{\partial S}+Q \frac{\partial M}{\partial Q}\right)\left(\begin{array}{cc}
-\frac{\partial^{2} M}{\partial S^{2}} & 0 \\
0 & \frac{\partial^{2} M}{\partial Q^{2}}
\end{array}\right)\left(\begin{array}{c}
d S^{2} \\
d Q^{2}
\end{array}\right) \\
= & \frac{\alpha^{2-2 N}\left[12 \alpha^{2} S-(2 N+1) Q^{2}\right]\left[12 \alpha^{2} S+(4 N-1) Q^{2}\right]}{2^{5+2 / N} \pi^{2} N^{3} S^{2+1 / N}} \\
& \times d S^{2}+\frac{\alpha^{2-2 N}\left(12 \alpha^{2} S+(4 N-1) Q^{2}\right)}{2^{2(N+1) / N} \pi^{2} N S^{1 / N}} d Q^{2} .
\end{aligned}
$$

The index $L$ denotes the curvature reproduced from the Legendre invariant metric.

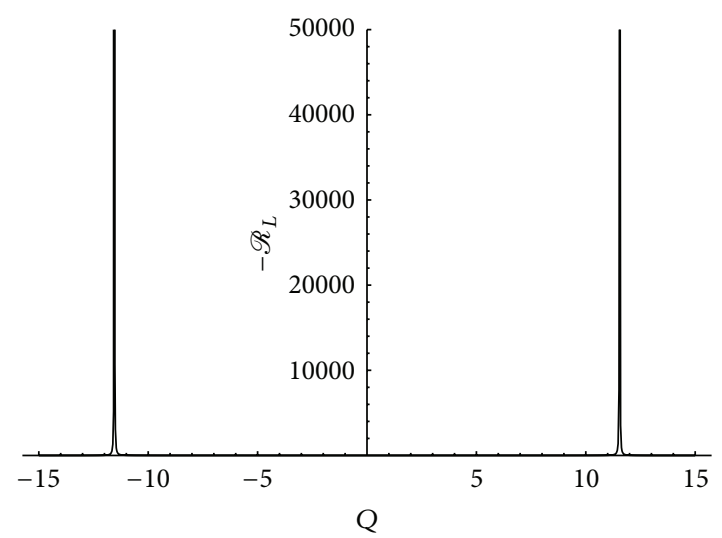

FIgURE 5: The negative unified geometric curvature $\mathscr{R}_{L}$ versus the charge $Q$ with $N=1, \alpha=\sqrt{3} / 3$, and $S=100$. The divergence points are at $Q= \pm 11.5470$, which are consistent with the divergence points of the heat capacity $C_{Q}$. The positive curvature region is at $|Q| \geq 20$ and it is not shown in this figure.

This diagonal metric reproduces the thermodynamic curvature $\mathscr{R}_{L}$, which turns out to be non-zero and the scalar curvature is

$$
\begin{aligned}
& \mathscr{R}_{L}= \frac{1924^{1 / N} \pi^{2} \alpha^{2 N} N S^{1+1 / N}}{\left[12 \alpha^{2} S-(2 N+1) Q^{2}\right]^{2}\left[12 \alpha^{2} S+(4 N-1) Q^{2}\right]^{3}} \\
& \cdot\left\{( 4 N - 1 ) Q ^ { 2 } \left[\left(N\left(4 N^{2}-6 N-3\right)-1\right) Q^{2}\right.\right. \\
&\left.\quad-12((N-5) N-2) \alpha^{2} S\right] \\
&\left.+144(N-1)^{2} \alpha^{4} S^{2}\right\} .
\end{aligned}
$$

The thermodynamic curvature vanishes at $Q^{2}=12 S \alpha^{2}$ when $N=1$, which are just the points of the first-order phase transition. It is shown that the diverge points are at $Q^{2}=12 \alpha^{2} S /(2 N+1)$, which implies that there exist second-order phase transitions at these points. This result exactly consists with that of the heat capacity (14). The detail behavior of $\mathscr{R}_{L}$ can be found in Figure 5, where the singularities are just the divergence points of the heat capacity $C_{Q}$. Now, we can see that the thermodynamic curvature $\mathscr{R}_{L}$ reproduced from the Legendre invariant metric (25) could give an exact description of the second-order phase transitions of a thermodynamics system. Beside this, we also expect that this unified geometry description may give more information about a thermodynamics system.

\section{Unified Geometry beyond Semiclassical Approximation}

In this section, we will discuss the unified geometry of the plane symmetric black hole beyond semiclassical approximation. With the idea, each quantity of the black hole will 


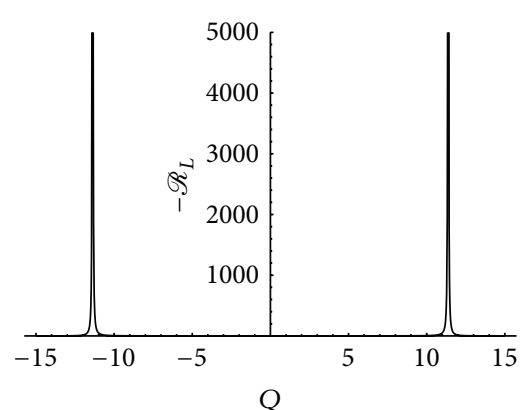

(a)

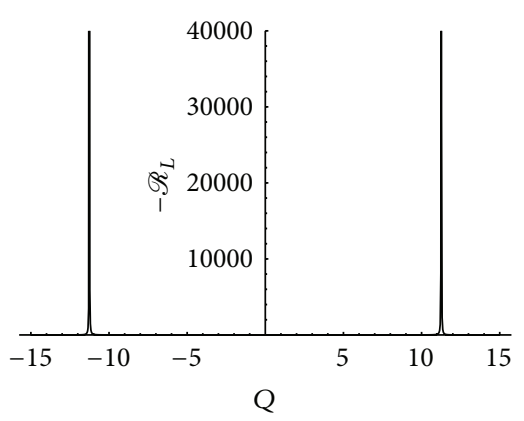

(b)

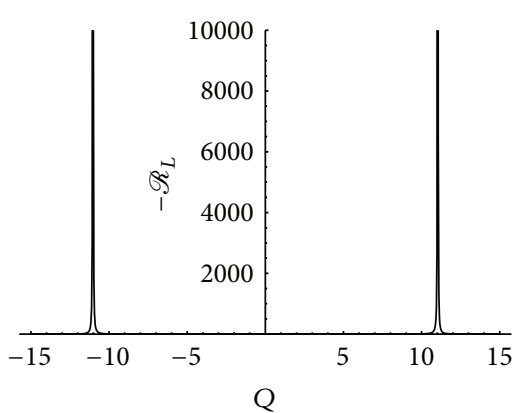

(c)

Figure 6: The negative unified geometric curvature $\mathscr{R}_{L}$ versus the charge $Q$, including the logarithmic correction with $N=1, \alpha=\sqrt{3} / 3$, and $S=100$. The parameter $\gamma$ is set to $1 / 2$ (left), $5 / 6$ (middle), and 3/2 (right), respectively. The divergence points are at $Q= \pm 11.3756, \pm 11.2613$, and \pm 11.0326 .

be modified accordingly. For general, we suppose that the corrected entropy is of the form

$$
S^{\prime}=S-\gamma \ln S .
$$

The parameter $\gamma$ is a constant. In fact, the origin of the logarithmic correction term can be accounted by the uncertainty principle or the tunneling method. With (27), the heat capacity (14) is also modified to

$$
C_{\mathrm{Q}}^{\prime}=\frac{2 N S(S-\gamma)(S-\gamma \ln S)\left(12 S \alpha^{2}-Q^{2}-12 \alpha^{2} \gamma \ln S\right)}{A_{2} Q^{2}-A_{1}},
$$

with

$$
\begin{gathered}
A_{1}=12 \alpha^{2}(S-\gamma \ln S)\left[(1+2 N \ln S) \gamma^{2}-2(N+1) S \gamma+S^{2}\right], \\
A_{2}=[2 N(1+\ln S)+1] \gamma^{2}-2(3 N+1) S \gamma+(2 N+1) S^{2} .
\end{gathered}
$$

The singular points of the heat capacity are determined by $A_{2} Q^{2}-A_{1}=0$ and are given by

$$
Q^{2}=\frac{A_{1}}{A_{2}}
$$

If $\gamma=0$, the singular points of the heat capacity will reduce to (14). Following Section 4, we obtain the curvature $\mathscr{R}_{L}^{\prime}$ :

$$
\mathscr{R}_{L}^{\prime}=\frac{h(S, Q, N, \gamma)}{K^{3}\left(A_{2} Q^{2}-A_{1}\right)^{2}},
$$

where $K=[4 N(S-\gamma \ln S)+\gamma-S] Q^{2}+12 \alpha^{2}(S-\gamma)(S-\gamma \ln S)$ and $h(S, Q, N, \gamma)$ is a complex function and we do not write it here. It is found that the divergence points for the heat capacity $C_{Q}^{\prime}$ and the curvature $\mathscr{R}_{L}^{\prime}$ consist with each other, which means that the curvature gives proper points, where second-order phase transitions take place. So, it is easy to summarize that the logarithmic correction term does not affect the unified geometry to depict the plane symmetry black hole's phase transitions.
Now, we would like to discuss how the geometry behaved as the parameter $\gamma$ takes different values. For simplicity, we turn back to the case $N=1$. The Legendre invariant metric for this case is

$$
g_{L}=\left(\begin{array}{cc}
-\frac{B C}{128 \pi^{2} S^{2}(S-\gamma \ln S)^{4}} & 0 \\
0 & \frac{B}{16 \pi^{2} S^{2}(S-\gamma \ln S)^{2}}
\end{array}\right),
$$

where $B=\left.K\right|_{N=1}$ and $C=\left.\left(A_{2} Q^{2}-A_{1}\right)\right|_{N=1}$. After some tedious calculations, we can obtain the curvature. The numerator of the curvature is a cumbersome expression and can not be written in a compact form. While the denominator of it is proportional to the determinant of the metric (32) and is given by

$$
D=B^{3} \cdot C^{2} .
$$

Fixing the parameters $\alpha$ and entropy $S$, the characteristic behavior of the curvature is depicted in Figure 6, where the parameter $\gamma$ is set to $1 / 2,5 / 6$ and $3 / 2$, respectively. The values of the charge $Q$ at the divergence points of the curvature $\mathscr{R}_{L}^{\prime}$ are given as

$$
Q= \pm 2 \sqrt{3} \alpha \sqrt{\frac{(S-\gamma \ln S)\left[(1+2 \ln S) \gamma^{2}-4 S \gamma+S^{2}\right]}{[2(1+\ln S)+1] \gamma^{2}-8 S \gamma+3 S^{2}}} .
$$

When $\Delta=3-2 \ln S \geq 0$, there are three points of $\gamma$ for the vanished charge $Q$ :

$$
\gamma_{1}=\frac{S}{\ln S}, \quad \gamma_{ \pm}=\frac{2 \pm \sqrt{3-2 \ln S}}{1+2 \ln S} S .
$$

In general, we consider $S \gg 1$, which leads to $\Delta<0$. So, the vanished charge $Q$ is only at $\gamma=\gamma_{1}$.

\section{Conclusion}

In this paper, we study the phase transitions and geometry structure of the plane symmetry black hole. The local 
thermodynamic stability of it is also discussed through the heat capacity $C_{Q}$. It is shown that there always exist locally thermodynamically stable phases and unstable phases for plane symmetric black hole due to suitable parameter regimes. The Weinhold geometry and the Ruppeiner geometry are obtained. The Weinhold curvature gives phase transition points, which correspond to that of the firstorder phase transition only at $N=1$, while the Ruppeiner one shows first-order phase transition points for arbitrary $N \neq 1$. Both of which give no information about the secondorder phase transition. Quevedo et al. first pointed out that the two geometry metrics are not Legendre invariant and they introduced a Legendre invariant metric, which can give a good description of various types of black hole thermodynamics. Considering the Legendre invariant, we obtain a unified geometry metric, which gives a correct behavior of the thermodynamic interaction and secondorder phase transition. Including the logarithmic corrected term, we study the geometry structure of the plane symmetry black hole. The result shows that the logarithmic correction term does not affect the unified geometry to depict the phase transitions. In this paper, we show that the unified geometry description gives a good description of the second-order phase transitions of the plane symmetry black hole. We also expect that this unified geometry description may give more information about a thermodynamic system.

\section{Acknowledgments}

This work was supported by the National Natural Science Foundation of China (Grant no. 11205074 and Grant no. 11075065) and the Huo Ying-Dong Education Foundation of the Chinese Ministry of Education (Grant no. 121106), and the Fundamental Research Funds for the Central Universities (no. lzujbky-2013-18 and no. lzujbky-2013-21).

\section{References}

[1] J. D. Bekenstein, "Black holes and entropy," Physical Review D, vol. 7, pp. 2333-2346, 1973.

[2] S. W. Hawking, "Particle creation by black holes," Communications in Mathematical Physics, vol. 43, no. 3, pp. 199-220, 1975.

[3] J. M. Bardeen, B. Carter, and S. W. Hawking, "The four laws of black hole mechanics," Communications in Mathematical Physics, vol. 31, pp. 161-170, 1973.

[4] P. C. W. Davies, "The thermodynamic theory of black holes," Proceedings of the Royal Society A, vol. 353, pp. 499-521, 1977.

[5] P. C. W. Davies, "Thermodynamics of black holes," Reports on Progress in Physics, vol. 41, p. 1313, 1977.

[6] P. C. W. Davies, "Thermodynamic phase transitions of KerrNewman black holes in de Sitter space," Classical and Quantum Gravity, vol. 6, no. 12, p. 1909, 1989.

[7] F. Weinhold, "Metric geometry of equilibrium thermodynamics," The Journal of Chemical Physics, vol. 63, no. 6, pp. 24792483, 1975.

[8] G. Ruppeiner, "Thermodynamics: a Riemannian geometric model," Physical Review A, vol. 20, no. 4, pp. 1608-1613, 1979.
[9] G. Ruppeiner, "Riemannian geometry in thermodynamic fluctuation theory," Reviews of Modern Physics, vol. 67, pp. 605-659, 1995, Erratum: Reviews of Modern Physics, vol. 68, p. 313, 1996.

[10] S. Ferrara, G. W. Gibbons, and R. Kallosh, "Black holes and critical points in moduli space," Nuclear Physics B, vol. 500, no. 1-3, pp. 75-93, 1997.

[11] R.-G. Cai and J.-H. Cho, "Thermodynamic curvature of the BTZ black hole," Physical Review D, vol. 60, no. 6, Article ID 067502, 1999.

[12] J. E. Åman, I. Bengtsson, and N. Pidokrajt, "Geometry of black hole thermodynamics," General Relativity and Gravitation, vol. 35, no. 10, pp. 1733-1743, 2003.

[13] D. A. Johnston, W. Janke, and R. Kenna, "Information geometry, one, two, three (and four)," Acta Physica Polonica B, vol. 34, no. 10, pp. 4923-4937, 2003.

[14] G. Arcioni and E. Lozano-Tellechea, "Stability and critical phenomena of black holes and black rings," Physical Review D, vol. 72, no. 10, Article ID 104021, 2005.

[15] J. Shen, R.-G. Cai, B. Wang, and R.-K. Su, “Thermodynamic geometry and critical behavior of black holes," International Journal of Modern Physics A, vol. 22, no. 1, pp. 11-27, 2007.

[16] J. E. Åman, I. Bengtsson, and N. Pidokrajt, "Flat information geometries in black hole thermodynamics," General Relativity and Gravitation, vol. 38, no. 8, pp. 1305-1315, 2006.

[17] J. E. Åman and N. Pidokrajt, "Geometry of higher-dimensional black hole thermodynamics," Physical Review D, vol. 73, no. 2, Article ID 024017, 2006.

[18] B. Mirza and M. Zamaninasab, "Ruppeiner geometry of RN black holes: flat or curved?" Journal of High Energy Physics, no. 6, 2007.

[19] J. E. Aman, N. Pidokrajt, and J. Ward, "On geometrothermodynamics of Dilaton black holes," EAS Publications Series, vol. 30, p. 279, 2008.

[20] J. E. Åman and N. Pidokrajt, "Ruppeiner geometry of black hole thermodynamics," EAS Publications Series, vol. 30, pp. 269-273, 2008.

[21] A. J. M. Medved, "A commentary on Ruppeiner metrics for black holes," Modern Physics Letters A, vol. 23, no. 26, pp. 21492161, 2008.

[22] Y. S. Myung, Y.-W. Kim, and Y.-J. Park, "Ruppeiner geometry and 2D dilaton gravity in the thermodynamics of black holes," Physics Letters B, vol. 663, no. 4, pp. 342-350, 2008.

[23] L. A. Gergely, N. Pidokrajt, and S. Winitzki, "Geometrothermodynamics of tidal charged black holes," European Physical Journal C, vol. 71, p. 1569, 2011.

[24] Y.-H. Wei, "Thermodynamic critical and geometrical properties of charged BTZ black hole," Physical Review D, vol. 80, no. 2, Article ID 024029, 2009.

[25] R. Biswas and S. Chakraborty, "The geometry of the higher dimensional black hole thermodynamics in Einstein-GaussBonnet theory," General Relativity and Gravitation, vol. 42, no. 5, pp. 1311-1322, 2010.

[26] T. Sarkar, G. Sengupta, and B. N. Tiwari, "Thermodynamic geometry and extremal black holes in string theory," Journal of High Energy Physics, no. 10, 2008.

[27] S. Bellucci and B. N. Tiwari, "On the microscopic perspective of black branes thermodynamic geometry," Entropy, vol. 12, no. 10, pp. 2097-2143, 2010.

[28] J. E. Aman, J. Bedford, D. Grumiller, N. Pidokrajt, and J. Ward, "Ruppeiner theory of black hole thermodynamics," Journal of Physics: Conference Series, vol. 66, Article ID 012007, 2007. 
[29] G. Ruppeiner, "Thermodynamic curvature and phase transitions in Kerr-Newman black holes," Physical Review D, vol. 78, no. 2, Article ID 024016, 2008.

[30] S. I. Vacaru, "Thermodynamic geometry and locally anisotropic black holes," http://arxiv.org/abs/gr-qc/9905053.

[31] H. Quevedo, "Geometrothermodynamics," Journal of Mathematical Physics, vol. 48, no. 1, Article ID 013506, 2007.

[32] H. Quevedo and A. Vazquez, "The geometry of thermodynamics," AIP Conference Proceedings, vol. 977, p. 165, 2008.

[33] H. Quevedo and A. Sánchez, "Geometric description of BTZ black hole thermodynamics," Physical Review D, vol. 79, no. 2, Article ID 024012, 2009.

[34] H. Quevedo, "Geometrothermodynamics of black holes," General Relativity and Gravitation, vol. 40, no. 5, pp. 971-984, 2008.

[35] J. L. Álvarez, H. Quevedo, and A. Sánchez, "Unified geometric description of black hole thermodynamics," Physical Review D, vol. 77, no. 8, Article ID 084004, 2008.

[36] H. Quevedo and A. Sánchez, "Geometrothermodynamics of asymptotically anti-de Sitter black holes," Journal of High Energy Physics, no. 9, 2008.

[37] H. Quevedo, A. Sanchez, and A. Vazquez, "Invariant geometry of the ideal gas," http://arxiv.org/abs/0811.0222.

[38] H. Quevedo and A. Sánchez, "Geometrothermodynamics of black holes in two dimensions," Physical Review D, vol. 79, no. 8, Article ID 087504, 2009.

[39] H. Liu, H. Lu, M. Luo, and K.-N. Shao, "Thermodynamical metrics and black hole phase transitions," Journal of High Energy Physics, no. 54, 2010.

[40] Q. J. Cao, Y. X. Chen, and K. N. Shao, "Black hole phase transitions in Hořava-Lifshitz gravity," Physical Review D, vol. 83, Article ID 064015, 2011.

[41] M. Akbar, H. Quevedo, K. Saifullah, A. Sanchez, and S. Taj, "Thermodynamic geometry of charged rotating BTZ black holes," Physical Review D, vol. 83, Article ID 084031, 2011.

[42] P. Chen, "Thermodynamic Geometry of the Born-Infeld-antide Sitter black holes," International Journal of Modern Physics A, vol. 26, p. 3091, 2011.

[43] S. W. Wei, Y. X. Liu, Y. Q. Wang, and H. Guo, "Thermodynamic geometry of black hole in the deformed Hořava-Lifshitz gravity," Europhysics Letters, vol. 99, Article ID 20004, 2012.

[44] G. Ruppeiner, "Thermodynamic curvature: pure fluids to black holes," Journal of Physics: Conference Series, vol. 410, Article ID 012138, 2013.

[45] K. Huang, Statistical Mechanics, John Wiley \& Sons, New York, NY, USA, 1963.

[46] L. D. Landau and E. M. Lifshitz, Statistical Physics, Pergamon, 1969.

[47] T. Sarkar, G. Sengupta, and B. N. Tiwari, "On the thermodynamic geometry of BTZ black holes," Journal of High Energy Physics, no. 11, 2006.

[48] R.-G. Cai and Y.-Z. Zhang, "Black plane solutions in fourdimensional spacetimes," Physical Review D, vol. 54, no. 8, pp. 4891-4898, 1996.

[49] A. S. Miranda, J. Morgan, and V. T. Zanchin, "Quasinormal modes of plane-symmetric black holes according to the AdS/CFT correspondence," Journal of High Energy Physics, no. 11, 2008.

[50] A.S. Miranda and V. T. Zanchin, "Quasinormal modes of planesymmetric anti-de Sitter black holes: a complete analysis of the gravitational perturbations," Physical Review D, vol. 73, Article ID 064034, 2006.
[51] X.-X. Zeng, Y.-W. Han, and Y. S. Zheng, "Hawking radiation from plane symmetric black hole covariant anomaly," Communications in Theoretical Physics, vol. 51, no. 1, pp. 187-189, 2009.

[52] R. Zhao, L. C. Zhang, and Y. Q. Wu, "Calculating entropy of plane symmetry black hole via generalized uncertainty relation," International Journal of Theoretical Physics, vol. 46, p. 3128, 2007.

[53] J. P. S. Lemos and F. S. N. Lobo, "Plane symmetric traversable wormholes in an anti-de Sitter background," Physical Review D, vol. 69, no. 10, Article ID 104007, 2004. 

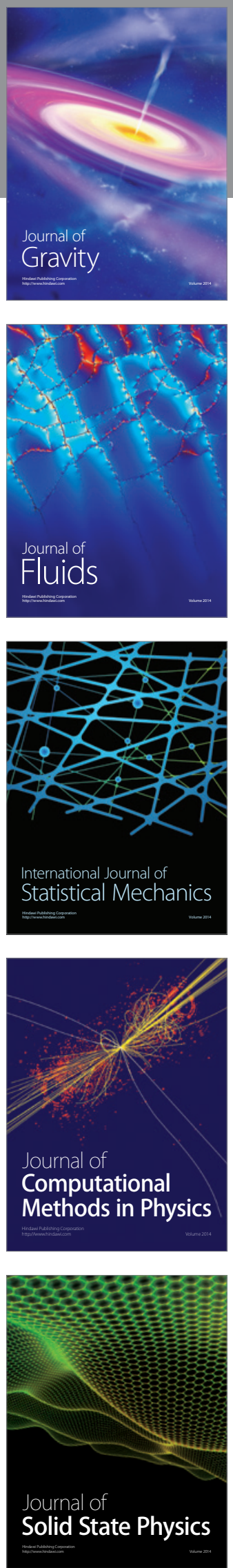

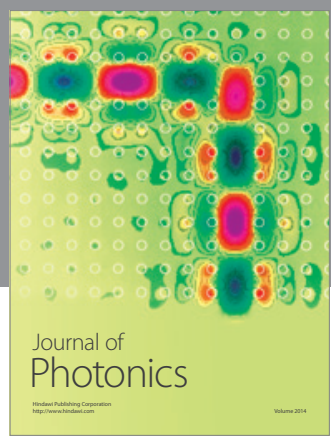

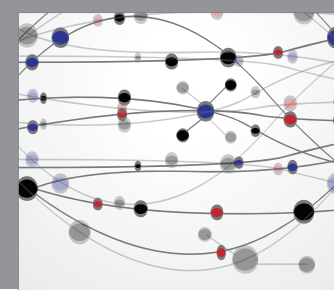

The Scientific World Journal

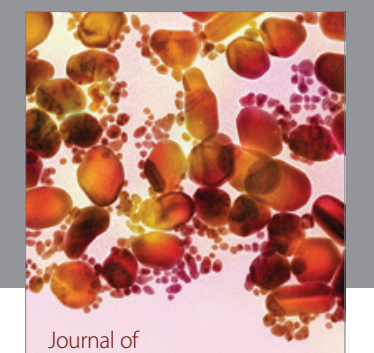

Soft Matter
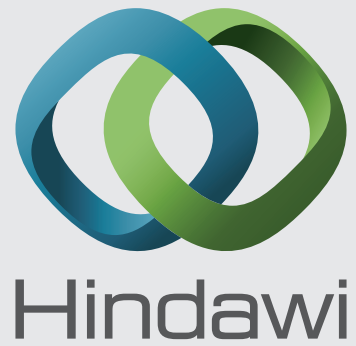

Submit your manuscripts at

http://www.hindawi.com
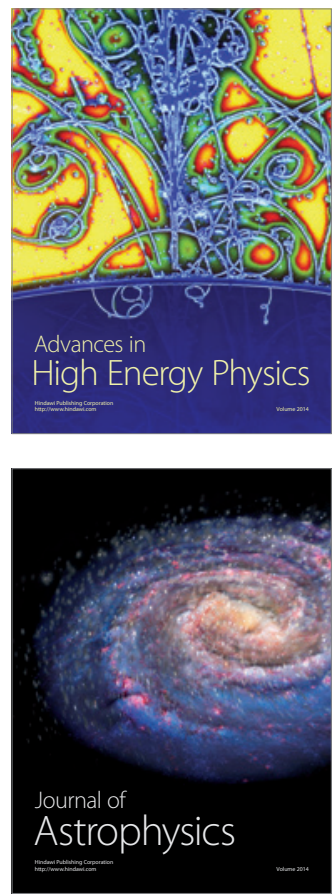
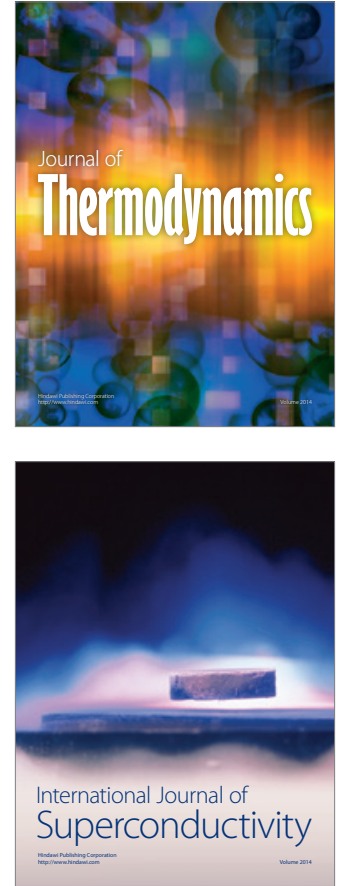
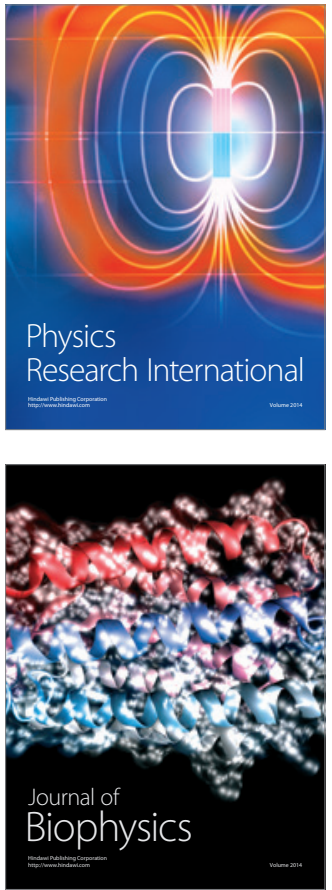
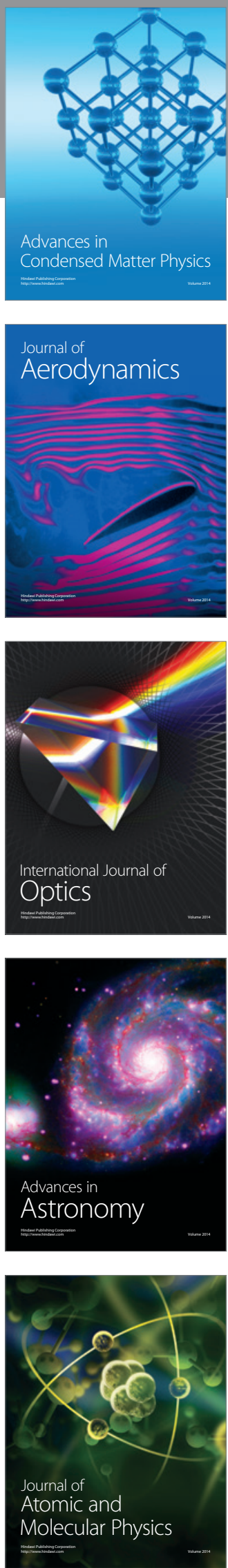